\title{
PET/MR Synchronization by Detection of Switching Gradients
}

\author{
Bjoern Weissler, Pierre Gebhardt, Christoph W. Lerche, Georgios M. Soultanidis, Jakob Wehner, \\ Dirk Heberling, and Volkmar Schulz
}

\begin{abstract}
The full potential of simultaneous Positron Emission Tomography (PET) and Magnetic Resonance Imaging (MRI) acquisition, such as dynamic studies or motion compensation, can only be explored if the data of both modalities is temporally synchronized. As such hybrid imaging systems are commonly realized as custom-made PET inserts for commercially available MRI scanner, a synchronization solution has to be implemented (depending on the vendor of the MRI system). In contrast, we demonstrate a simple method for temporal synchronization, which does not require a connection to the MRI. It uses the normally undesired effect of induced voltages on the PET electronics from switching MRI gradients. The electronic circuit needs very few components and the gradient pick-up coils are made from PCB traces and vias on the PET detector boards. Neither programming the MRI nor any physical connection to the MR scanner is needed, thus avoiding electromagnetic compatibility problems. This method works inherently with most MRI sequences and is a vendor- independent solution. A characterization of the sensors in an MRI scanner showed that the MRI gradients are detected with a precision of $120 \mu \mathrm{s}$ (with the current implementation). Using different trigger thresholds, it is possible to trigger selectively on certain MRI sequences, depending on their gradient slew rate settings. Timings and pulse diagrams of MRI sequences can be recognized from the generated
\end{abstract}

Manuscript received August 29, 2014; revised January 24, 2015; accepted April 26, 2015. Date of current version June 12, 2015. This work was part of the EU FP7 project HYPERImage, Grant Agreement 201651, and the EU FP7 project SUBLIMA, Grant agreement 241711. It was co-funded by the German federal state North Rhine Westphalia (HighTech. NRW) and the European Union (European Regional Development Fund: Investing In Your Future) in the ForSaTum project (grant z0903ht014 g), and the Centre of Excellence in Medical Engineering funded by the Wellcome Trust and EPSRC under grant WT $088641 / \mathrm{Z} / 09 / \mathrm{Z}$.

B. Weissler is with the Clinical Application Research Department, Philips Research, 52074 Aachen, Germany, and also with the Department of Physics of Molecular Imaging Systems, Institute of Experimental Molecular Imaging, RWTH Aachen University, 52074 Aachen, Germany (e-mail: bjoern.weissler@philips.com).

P. Gebhardt is with the Division of Imaging Sciences and Biomedical Engineering, King's College London, London SE1 7EH, U.K.

C. W. Lerche was with the Oncology Solutions Department, Philips Research, Eindhoven $5656 \mathrm{AE}$, The Netherlands.

G. M. Soultanidis was with the Division of Imaging Sciences and Biomedical Engineering, King's College London, London SE1 7EH, U.K.

J. Wehner is with the Department of Physics of Molecular Imaging Systems, Institute of Experimental Molecular Imaging, RWTH Aachen University, 52074 Aachen, Germany.

D. Heberling is with the Institute of High Frequency Technology, RWTH Aachen University, 52074 Aachen, Germany.

V. Schulz is with the Department of Physics of Molecular Imaging Systems, Institute of Experimental Molecular Imaging, RWTH Aachen University, 52074 Aachen, Germany, and also with the Clinical Application Research Dpartment, Philips Research, 52074 Aachen, Germany (e-mail: schulz@pmi.rwth-aachen. de).

Color versions of one or more of the figures in this paper are available online at http://ieeexplore.ieee.org.

Digital Object Identifier 10.1109/TNS.2015.2427995 data. The method was successfully used for temporal alignment between PET and MRI in an MRI-based PET-motion-compensation application.

Index Terms-Magnetic resonance imaging, molecular imaging, PET-MR, positron emission tomography, synchronization.

\section{INTRODUCTION}

$\mathbf{T}$ HE advantages of simultaneous Positron Emission Tomography (PET) and Magnetic Resonance Imaging (MRI) image acquisition are not only shorter scan times, smaller system footprints, and less image registration problems. Having both data from exactly the same time enables new pplications, such as dynamic studies or motion compensation [1]. The latter will be used in this paper as an example application, showing the need for time synchronization, and demonstrating how the detection of switching gradients can achieve that.

The motivation behind PET motion compensation is that regions in the body moving during a long PET measurement are reconstructed with motion artefacts or become blurry (thus reducing the detectability of small lesions). One idea is to track movements and deformation with the MRI, while the PET data is acquired [2]. The PET data can be corrected subsequently or gated into separate bins (e.g. for CINE reconstructions, showing periodic movements). To study these possibilities, a polyvinyl alcohol (PVA) cryogel phantom was built. It can be deformed elastically to model the movements in a body caused by breathing [3]. Since breathing is normally an irregular pattern, the phantom is deformed according to previously recorded human breathing pattern [4]. Uncorrected, this results in a blurred image as shown in Fig. 1.

The phantom was imaged simultaneously with a PET/MR system (Fig. 2) that is realized as a preclinical PET/RF insert [5] for a clinical $3 \mathrm{~T}$ MRI scanner (Achieva, Philips, The Netherlands). An echo planar imaging (EPI) MRI sequence was used to scan repetitively for 9 minutes with a temporal resolution of $0.601 \mathrm{~s}$ (spatial resolution: $0.81 \mathrm{~mm} \times 0.81 \mathrm{~mm} \times 1.2 \mathrm{~mm}, \mathrm{TR} / \mathrm{TE}$ : $2.4 \mathrm{~ms} / 0.85 \mathrm{~ms}, 20^{\circ}$ flip angle).

From the resulting 900 images, a 1-D navigator was extracted, and the movement was calculated by the correlation to a reference navigator. The result, as displayed in Fig. 3, has one substantial problem before it can be used to gate PET data: although the timing of the MRI sequence (once it started) is known precisely, the actual moment in time, when it starts is depending on multiple uncontrollable factors: firstly the 

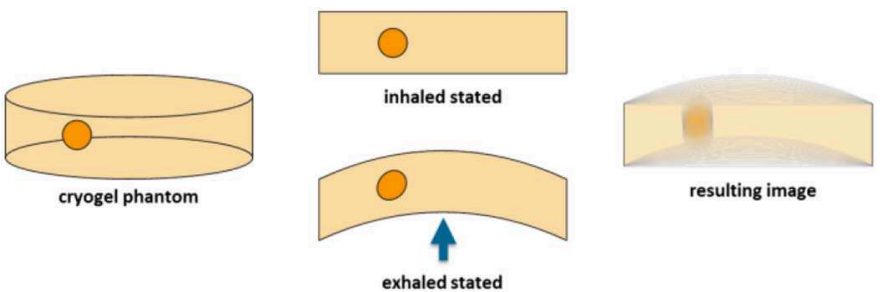

resulting image

exhaled stated

Fig. 1. Sketch of the PVA cryogel phantom with a hot region (left). With breathing deformation applied during PET data acquisition (middle), the resulting image is blurred (right).

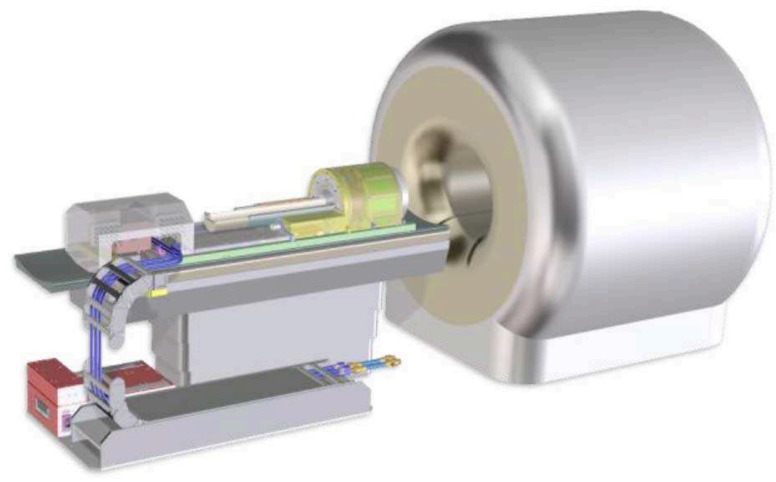

Fig. 2. 3D drawing of the PET/RF insert "Hyperion I" placed on the patient table of a clinical MRI scanner.

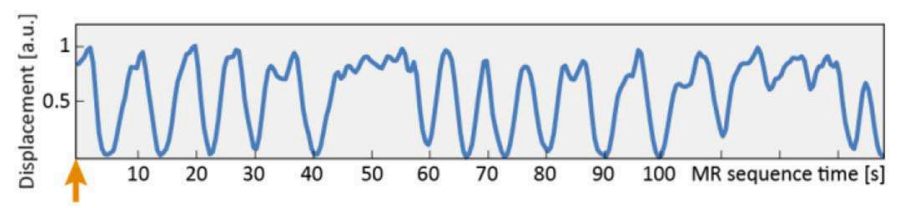

Fig. 3. Normalized displacement in a 1D navigator of the measured phantom. The phantom was deformed with a previously recorded human breathing rhythm. The MR sequence time is not synchronized to the PET system time.

operator clicking "start scan" in the graphical user interface of the MRI console. Then the time taken to calculate the sequence and to send it to the real-time control of the scanner. Subsequently the hardware has to be brought into the correct operating points, which depends on the sequence (e.g. whether the cryopump needs to be switched off) and on the history (e.g. whether components still need to warm up). The length of the now following preparation phase of the MRI scan is also undetermined, especially when some iterative optimizations such as automatic shimming are included.

The only currently commercially available combined PET/MRI scanner inserts a synchronization tag into the PET list-mode data, marking the beginning of the MRI sequence [6]. Most systems are - just as the one used - custom-made PET inserts for (relatively closed) standard MRI systems. If the systems are not inherently combined (therefore being completely separate machines), there are no direct temporal synchronization methods implemented. In that case, output trigger signals have to be obtained from the MRI and forwarded to the PET system, which needs the respective inputs [7]. Depending on the systems used, this might require hardware and software modifications and can introduce the risk of image degradation due to additional cabling.

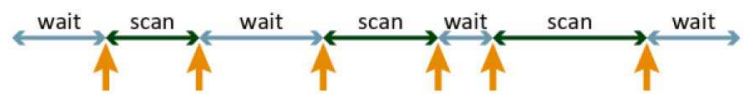

Fig. 4. Breath gated MRI sequence: The MRI decides with a breathing monitor when to scan. The PET system needs to know beginning and end of each scanning period.

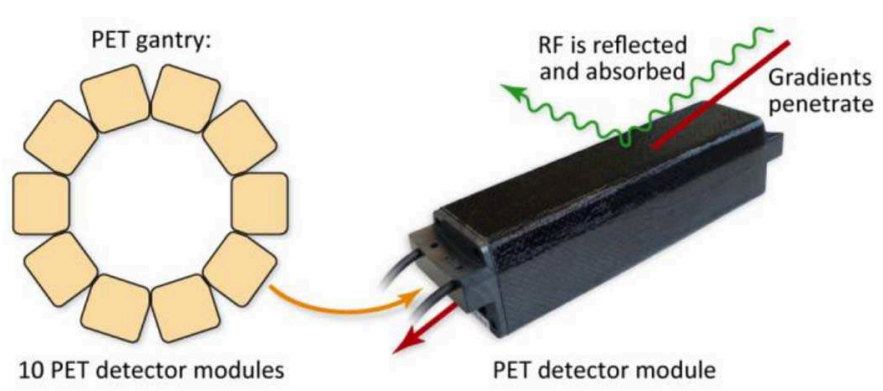

Fig. 5. The PET gantry (cross section shown on the left) is composed of ten individual PET detector modules (right). They are screened against RF fields, but low-frequent gradient fields penetrate the shielding.

\section{A. Aim}

The aim of this paper is to synchronize PET and MRI without any modifications or connections to the MRI. It will be realized by the detection of the switching MRI gradients.

\section{B. Requirements}

Although for the presented example of human breathing, the needed precision is roughly $300 \mathrm{~ms}$ (time resolution of the MRI scan divided by two), the precision should be in the range of milliseconds, so it can be used with mouse breathing and cardiac cycles. When the MRI sequence timing is known precisely, only one synchronization step in the beginning of the sequence is needed (when sampling of the k-space begins).

In rather advanced scenarios, it might be useful to synchronize more often during the MRI scan. For instance, when the MRI can switch seamlessly between two interleaved sequences [8]. An example for this (e.g. for motion compensation) would be a navigator sequence mixed with a normal imaging sequence. In that case, one would like to know the beginning of each navigator sequence to assign a time to the detected motion. It is thus important to differentiate between the two sequences. There are cases in which the timing of the MRI sequence is not predefined but determined during its execution, e.g. by a breathing monitor. In that situation, one might want to gate the PET data accordingly, and thus the beginning and end of MRI activity has to be detected (Fig. 4).

\section{Detection of Switching MRI GRAdients}

When PET detectors are brought into the bore of MRI scanners, they are normally shielded against the intense Radio Frequency (RF) fields (here: $128 \mathrm{MHz}$ at $3 \mathrm{~T}$ ). The switching of the MRI gradients, on the other hand, has a much lower frequency in the $\mathrm{kHz}$ range. Thus, the gradient fields penetrate the thin shielding (here: $18 \mu \mathrm{m}$ copper [5] or multiple layer of carbon fibers [9]) and induce voltages in conductive loops of the electronic circuits (Fig. 5). 


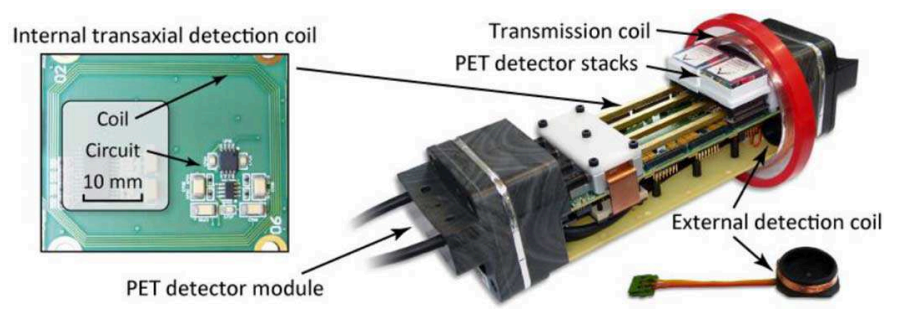

Fig. 6. Internal transaxial detection coil and the electronic circuit for two channels (left). An external detection coil is connected to the second channel for lab experiments, picking up test signals from a transmission coil (right).
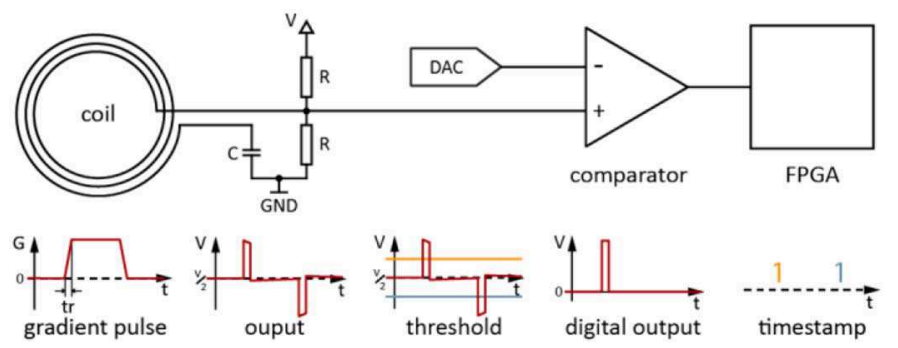

Fig. 7. Gradient switching detection circuit (from left to right): pick-up coil, AC-coupling to voltage divider, threshold-defining DAC, comparator, and time stamp generation in the FPGA. The lower row indicates the shape of the gradient intensity (left) and the signal over time at each stage of the circuit.

This effect is often reported as a degradation of PET energy resolution [10], a deterioration of time resolution [11], or as drops in count rates [12]. Therefore, the Printed Circuit Boards (PCBs) of the detector modules are carefully designed to minimize induced voltages - especially all conductive loops are kept as small as possible.

In contrast to these endeavors, we deliberately implemented two coils from PCB traces and vias on the mainboard of each PET detector module [13] (see Fig. 7) to pick up induced voltages from the switching gradients. The transaxial detection coil on the bottom of the PCB has 5 windings around an area of $36 \mathrm{~mm} \times 28 \mathrm{~mm}$. The axial coil has 15 windings, made from traces on the top and on the bottom side of the PCB, connected with vias to form the loops (the area is $48 \mathrm{~mm} \times 2 \mathrm{~mm}$ ). The two coils are connected in series, so that voltages induced by changing fields of the Z-gradient add up. Additionally, an external coil can be plugged into a connector for a second detector channel. Such a coil with 50 windings and a diameter of $23 \mathrm{~mm}$ was used in early lab experiments, as this coil is sensitive enough to pick up signals from a transmission coil directly connected to signal generator (all experiments presented in this paper employed only the PCB-trace-coils).

The coils are connected to a detection circuit (see Fig. 7). A voltage is induced by the changing flux through the windings when the gradients of the MRI switch. The coil is AC-coupled to a voltage divider. It shifts positive and negative pulses into the positive voltage range that can be used by standard electronics. A software-controlled Digital-to-Analog Converter (DAC) sets a threshold voltage, which is then compared against the voltage at the voltage divider by a comparator). The comparator has a $4 \mathrm{mV}$ hysteresis to suppress triggers on noise. The output is 1-bit digital signal and the firmware [14] in the Field Programmable

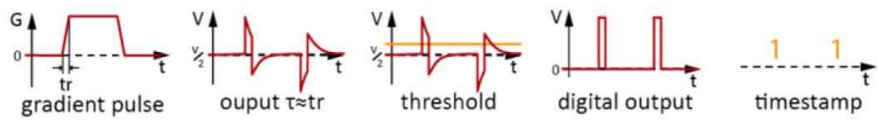

Fig. 8. Voltages over time in the gradient detection circuit with $\tau \approx \mathrm{t}_{\mathrm{r}}$.

Gate Array (FPGA) generates a message with a PET timestamp when this signal changes.

The simulated (SPICE) voltage curves over time in Fig. 7 visualize that only gradient slopes in one direction (here: the positive edge) would be detected, as the negative slopes would never pass the positive threshold. The negative slopes can also be detected if the AC-coupling capacitor $\mathrm{C}$ and the resistors $\mathrm{R}$ in the voltage divider are chosen in such a way that the time constant of the RC-circuit $\tau$ is close the rise time $\mathrm{t}_{\mathrm{r}}$ of the gradients. In that case, the capacitor is already significantly charged (or discharged) during the rise time. Once the rise time is over, no voltage is induced any more, and the voltage at the voltage divider is now defined by the built up charge in the capacitor (see Fig. 8). Therefore, a negative slope results in a positive signal after the voltage divider and can thus also be detected with a positive threshold.

The firmware is designed to be very versatile as many parameters can be software-controlled: it counts until a changeable number of triggers are reached, gives information about the first and the last detected trigger, and it has timeout settings in the range from one time bin up to more than 20 seconds. The FPGA sends timestamps (time bin width is currently $327.68 \mathrm{mi}-$ croseconds) for the first and the last detected trigger (which is needed in case the end of MRI activity is to be determined, see Fig. 4). Furthermore, the trigger edge (rising or falling) can be software-controlled (but is kept rising for all presented experiments).

An alternative to pick-up coils could be Hall-effect-based or magnetoresistive sensors that are also available as compact integrated circuits. Since these are also sensitive to static magnetic fields, one needs to pay attention to the dynamic range of the devices and/or to the spatial orientation with respect to the field.

\section{MeAsurement Methods}

\section{A. Sensor Characterization}

The gradient sensors were characterized on the second generation of the PET/RF insert "Hyperion $\mathrm{II}^{\mathrm{D}}$ " [15], [16], in which the gradient detection circuits are built in identically to "Hyperion I" [5], [17]. All PET detector modules have the same sensor circuits using only the build-in coils made from PCB traces. The MRI system is a Philips 3T Achieva MRI with a Quasar Dual gradient system. The two gradient amplifiers are connected in series (mode 1), which allows higher slew rates instead of higher gradient strength $(200 \mathrm{mT} / \mathrm{m} / \mathrm{msec}, 40 \mathrm{mT} / \mathrm{m})$.

A patched MRI software allows creating single gradient pulses with a known direction, strength, duration, rise time, and slew rate.

1) Threshold scan: The slew rate for the Z-gradient is set the highest slew rate possible $(200 \mathrm{mT} / \mathrm{m} / \mathrm{ms})$. The exact gradient waveform and a sketch of the fields produced by the Z-gradient are shown in Fig. 10 (top left). The trigger thresholds are varied 
TABLE I

Detailed SEQuence Information and Results for the SElective Triggering TeSts

\begin{tabular}{|c|c|c|c|c|c|c|c|c|c|}
\hline Sequence & Gradient Mode & $T R / T E$ & Scan Time & $\begin{array}{l}\text { Gradient } \\
\text { Strength }^{a}\end{array}$ & $\begin{array}{c}\text { Rise } \\
\text { Time }^{a}\end{array}$ & Slew Rate ${ }^{a}$ & $\begin{array}{l}\text { Trigger } \\
\text { at } 0 \mathrm{mV}\end{array}$ & $\begin{array}{c}\text { Trigger } \\
\text { at } 42 \mathrm{mV}\end{array}$ & $\begin{array}{c}\text { Trigger } \\
\text { at } 58 \mathrm{mV}\end{array}$ \\
\hline $\mathrm{T}_{1} \mathrm{w} \mathrm{aTSE}^{\mathrm{b}}$ & regular & $611 \mathrm{~ms} / 20 \mathrm{~ms}$ & $2: 09 \mathrm{~min}$ & $10 \mathrm{mT} / \mathrm{m}$ & $600 \mu \mathrm{s}$ & $17 \mathrm{mT} / \mathrm{m} / \mathrm{ms}$ & yes & - & - \\
\hline $\mathrm{T}_{1} \mathrm{w}_{\mathrm{TSE}}^{\mathrm{b}}$ & default & $612 \mathrm{~ms} / 20 \mathrm{~ms}$ & 2:09 $\min$ & $21 \mathrm{mT} / \mathrm{m}$ & $210 \mu \mathrm{s}$ & $99 \mathrm{mT} / \mathrm{m} / \mathrm{ms}$ & yes & yes & - \\
\hline Single-shot EPI ${ }^{c}$ & regular & $120 \mathrm{~ms} / 53 \mathrm{~ms}$ & $1.1 \mathrm{~s}$ & $10 \mathrm{mT} / \mathrm{m}$ & $600 \mu \mathrm{s}$ & $17 \mathrm{mT} / \mathrm{m} / \mathrm{ms}$ & yes & - & - \\
\hline Single-shot EPI ${ }^{c}$ & default & $56 \mathrm{~ms} / 23 \mathrm{~ms}$ & $508 \mathrm{~ms}$ & $21 \mathrm{mT} / \mathrm{m}$ & $210 \mu \mathrm{s}$ & $100 \mathrm{mT} / \mathrm{m} / \mathrm{ms}$ & yes & yes & - \\
\hline Single-shot EPI ${ }^{c}$ & maximum & $42 \mathrm{~ms} / 16 \mathrm{~ms}$ & $379 \mathrm{~ms}$ & $31 \mathrm{mT} / \mathrm{m}$ & $156 \mu \mathrm{s}$ & $198 \mathrm{mT} / \mathrm{m} / \mathrm{ms}$ & yes & yes & yes \\
\hline
\end{tabular}

a) Data shown for the Z-gradient in each echo

b) Echo train length: 6, Field of View: $160 \mathrm{~mm} \times 160 \mathrm{~mm}$, Voxel Size: $0.5 \mathrm{~mm} \times 0.5 \mathrm{~mm} \times 2 \mathrm{~mm}$

c) Echo Train Length: 64, Field of View: $160 \mathrm{~mm} \times 160 \mathrm{~mm}$, Voxel Size: $2.5 \mathrm{~mm} \times 2.5 \mathrm{~mm} \times 4 \mathrm{~mm}$

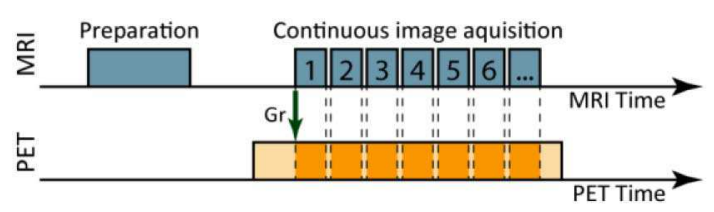

Fig. 9. Time diagram for motion compensation example: MRI preparation phase was executed upfront. Then, PET and MRI image acquisition were subsequently started. The first detected gradient is used for synchronization (Gr, green arrow). Since the MR image acquisition timing is known, the PET list-mode data can be linked to the MR images.

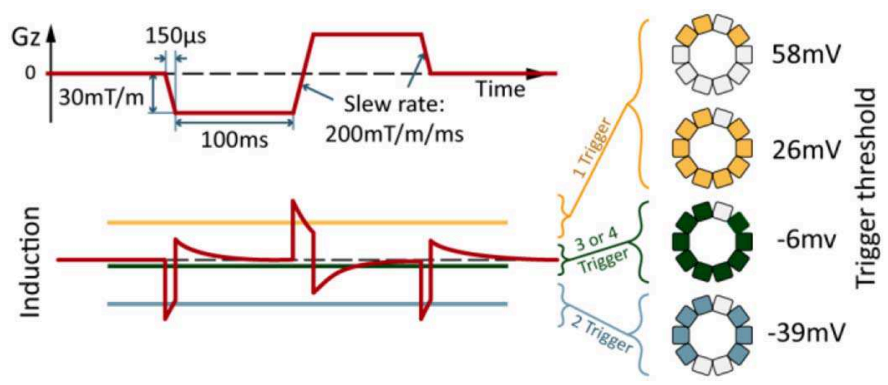

Fig. 10. Gradient waveform and simulated voltage at the input of the comparator (left) and the resulting trigger (right).

from $+80 \mathrm{mV}$ to $-80 \mathrm{mV}$ (relative to the equilibrium of the voltage divider) and the location and number of sensors triggered are evaluated.

2) Slew Rate Scans: By changing the rise time, the slew rate of the gradients is varied, and the lowest trigger threshold is determined at which at least one sensor triggers. Furthermore, the three gradient directions $\mathrm{X}, \mathrm{Y}$, and $\mathrm{Z}$ are tested separately.

In a separate experiment, the slew rate are varied in steps of $10 \mathrm{mT} / \mathrm{m} / \mathrm{ms}$, and the number of gradient sensors triggering are plotted for different trigger thresholds.

3) Time Accuracy and Precision: A 13-minute-long standard $\mathrm{T}_{2}$ weighted $\left(\mathrm{T}_{2} \mathrm{w}\right)$ spin echo sequence with a long repetition time, $\mathrm{TR}=2.4 \mathrm{~s}(\mathrm{TE}=50 \mathrm{~ms})$, and a slew rate of $59 \mathrm{mT} / \mathrm{m} / \mathrm{ms}$ (unchanged setting with normal Peripheral Nerve Stimulation (PNS) level) is executed. The threshold is left at the default value of $0 \mathrm{mV}$ and the number of triggers for a detector module is plotted over time. This sequence generates echoes with a time difference of exactly TR. Since TR is very long, the gradient triggers can easily be assigned to the different repetitions. The error in detection timing is calculated as the time difference between the first detected gradient of each repetition minus the repetition time TR.

\section{B. Impact on MR Image Quality}

When hardware is placed into the bore of the MRI system, it can directly interact with its three types of fields: RF-, gradient-, and static magnetic field $\left(\mathrm{B}_{0}\right)$. An interaction with the RF system is unlikely, since the sensor circuits are placed inside the RF-shielded housings. The gradients do interact with the sensor, as they induce voltages in the sensor coil. Since there is a resistor (in this case $1 \mathrm{kOhm}$ ) in series with the coil (see schematic in Fig. 7), the currents are more than three magnitudes lower than eddy currents in the ground planes of the PCB, and the influence on the gradient system is thus negligible.

The electronic components needed to build the electronic circuit often contain magnetic materials. In their vicinity, the homogeneity of the $\mathrm{B}_{0}$ field is distorted. To measure the effect, all 13 electronic components (for the used dual-channel sensor circuit, see Fig. 6, left) are placed on a homogeneous coronal body phantom. $\mathrm{A} \mathrm{B}_{0}$ field map of the phantom is generated on a $3 \mathrm{~T}$ system, and the range of the distortion is determined [5].

\section{Advanced Applications}

1) Selective Triggering: Using a DAC to change the threshold level (instead of a fixed two-resistor voltage divider) should enable triggering selectively on certain MRI sequences. The maximum slew rate of a sequence can be set manually or by changing gradient mode settings: Whereas "maximum" uses the maximum available gradient strength and slew rate (for fast scans), "regular" restricts the value (to achieve a better signal to noise ratio). The "default" setting chooses balanced values in between. These gradient modes are used for two different example MRI sequences: A Turbo Spin Echo (TSE) sequence and a single-shot EPI sequence (sagittal single slice, no preparation phases-see Application Example). Details of the sequences are shown in Table I. The trigger thresholds are set to the standard value of $0 \mathrm{mV}$, to $42 \mathrm{mV}$, and to $58 \mathrm{mV}$. For each combination it is recorded whether the sensors trigger or not.

2) Recognition of sequences: To test, whether an MRI sequence can be recognized in the data from the sensors, a Multiple Fast Field Echo (m-FFE) sequence was chosen, as it has an intuitive standard usage of gradients and most timings can be controlled manually. Here, an echo train of five echoes, each $\mathrm{TE}_{\text {extension }}=10 \mathrm{~ms}$ apart with a $\mathrm{TE}$ of $50 \mathrm{~ms}$ before the first echo is programmed. The repetition time $\mathrm{TR}=2000 \mathrm{~ms}$ is relatively long, compared the $\mathrm{TE}$ and $\mathrm{TE}_{\text {extension, }}$, so that all timings can be distinguished. The trigger threshold is $0 \mathrm{mV}$. 
3) Recognition of sequence phases: A scout scan sequence (gradient echo, TR/TE $=9.9 \mathrm{~ms} / 1.25 \mathrm{~ms}$ ), normally used as a first scan to plan other detailed MRI scans, is used in this experiment. As every other standard sequence, it consists of a preparation phase, followed by the k-space filling (for three orthogonal stacks with seven, five, and five images each). The preparation phase itself has different parts, like $\mathrm{f}_{0}$ determination, power optimization, and RF noise level determination. The latter part is very distinct, as it uses crusher gradients (with a rise time of $389 \mu \mathrm{s}$ and slew rate of $25 \mathrm{mT} / \mathrm{m} / \mathrm{ms}$ ) in all directions to diphase residual net magnetizations. They are followed by $150 \mathrm{~ms}$ of absolute silence, when the system measures the RF noise. In this sequence, the noise level determination is executed three times, which shall be recognized in the data from the gradient sensors. The trigger threshold is the default $0 \mathrm{mV}$.

\section{Application Example}

The synchronization circuits are used to determine the beginning of the motion detection sequence, which was presented in the introduction of this paper. Fig. 9 shows a time diagram of the sequence.

The preparation phase of the MRI sequence is executed separately before the image acquisition was started. After that, the gradient detection circuit are enabled and the threshold voltage is set to the standard (highly sensitive) $0 \mathrm{mV}$. Once, the PET data acquisition begins, the MRI sequence is started. Since there is no further preparation phase, the first overall detected gradient is directly the start of the imaging sequence. The generated PET time stamp is thus the time stamp of the first MR image (and therefore the time stamp of the first extracted motion navigator). The MRI sequence is programmed as a dynamic scan, acquiring the 900 images exactly $601 \mathrm{~ms}$ apart. The PET time stamps of the following MR images can be calculated and no further synchronization is needed for this scan.

\section{RESUlts}

\section{A. Sensor Characterization}

1) Threshold Scan: Fig. 10 shows the gradient waveform over time, the voltage at the input of the comparators (simulated), and how many triggers are counted in which detector module for four different threshold voltages. Threshold values larger than $+74 \mathrm{mV}$ disable the sensors, since no gradients are detected. With high, positive trigger thresholds, the first detector modules on the top of the insert count one trigger (except for one module, which seems to have a defect circuit). Reduction of the threshold results in detected gradients also in lower modules. When the threshold voltage reaches $0 \mathrm{mV}$, three or four triggers are counted. Negative thresholds below a certain level (blue line in the graph of the simulated voltage at the comparator in Fig. 10) yield to two triggers per module. When the threshold voltage is further lowered, modules at the bottom stop to trigger. At threshold voltages lower than $-70 \mathrm{mV}$, no sensor triggers any more.

2) Slew Rate Scans: The lowest slew rate of the gradients, where the first gradient sensor triggers, is plotted over different threshold voltages in Fig. 11. From lower negative thresholds
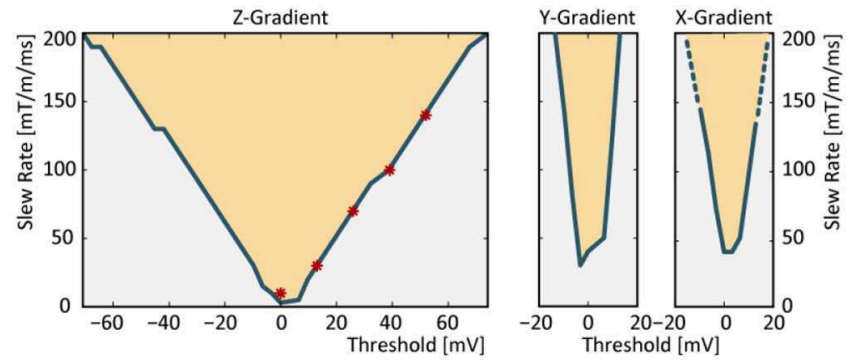

Fig. 11. Lowest slew rate at which the first sensor triggers over threshold voltage. The scales are the same for the Z-gradient (left), and Y- and X-gradients (right). The red stars show data from the measurements of Fig. 12.

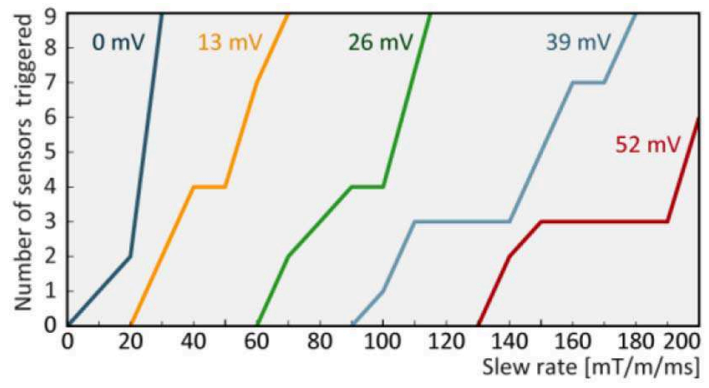

Fig. 12. Number of sensors (modules) triggered at increased slew rates of the Z-gradient for different threshold voltages.

(for the Z-gradient greater than $-70 \mathrm{mV}$ ) to $0 \mathrm{mV}$, the needed slew rate to trigger decreases almost linearly. For positive thresholds, it also decreases (almost) linearly with the same slope. At a gradient strength of $30 \mathrm{mT} / \mathrm{m}$, the first sensor triggers already at a slew rate of $3 \mathrm{mT} / \mathrm{m} / \mathrm{ms}(0 \mathrm{mV}$ threshold level). Very low gradient strengths result into very short pulses, because the targeted gradient strength is reached very fast. In that case, a higher slew rate is needed: at a gradient strength of $1 \mathrm{mT} / \mathrm{m} / \mathrm{ms}$ a slew rate of $15 \mathrm{mT} / \mathrm{m} / \mathrm{ms}$ triggers the first sensor reliably. The sensors are much less sensitive to gradients in $\mathrm{X}$ - and Y-direction: for the $\mathrm{X}$-gradient, the lowest slew rate is $40 \mathrm{mT} / \mathrm{m} / \mathrm{ms}$, and for the $y$-gradient it is $30 \mathrm{mT} / \mathrm{m} / \mathrm{ms}$. Also the threshold voltage, where the maximum available slew rate is needed to trigger, is lower: for the Y-gradient it is $\pm 15 \mathrm{mV}$. The same seems to be true for the X-gradient, although with the software patch it could only be driven to $140 \mathrm{mT} / \mathrm{m} / \mathrm{ms}$ (graph extrapolated as dotted lines).

The total number of sensors (and thus modules at different locations), that triggered for different threshold levels over the slew rate (in steps of $10 \mathrm{mT} / \mathrm{m} / \mathrm{ms}$ ) are shown in Fig. 12.

3) Time Accuracy and Precision: Fig. 13 depicts that during the $\mathrm{T}_{2} \mathrm{w}$ spin echo sequence, the sensors trigger very regularly: messages (with multiple counted triggers) are generated for each echo. The mean time difference to TR is $(20 \pm 120) \mu \mathrm{s}$, which can be interpreted as accuracy and precision of the measurement.

\section{B. Impact on MR Image Quality}

The $\mathrm{B}_{0}$-distortion, caused by the electronic circuit, is shown in Fig. 14. At a distance of about $25 \mathrm{~mm}$ in all directions, the disturbance is not noticeable any more. 

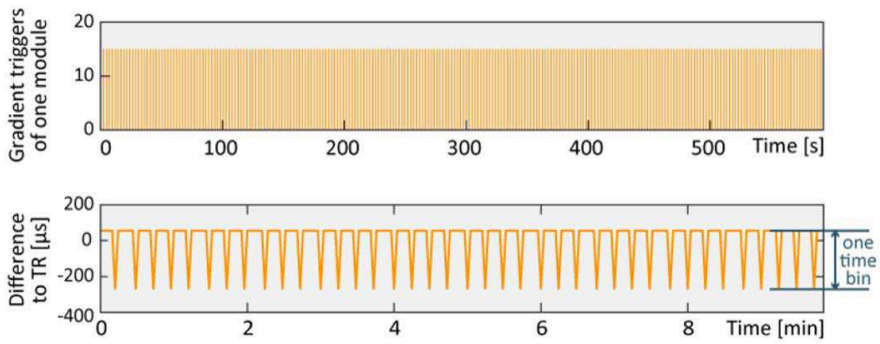

Fig. 13. Timing of gradient triggers for one module (top) and the difference to the repetition time $\mathrm{TR}=2.4 \mathrm{~s}$ (bottom) of the MRI sequence ( 9 of 13 minutes).
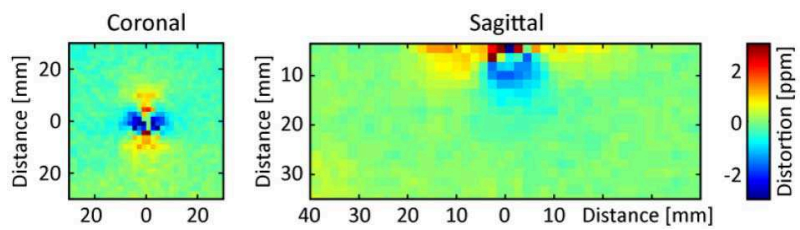

Fig. 14. $B_{0}$ field maps, showing the distortion caused by the electronic circuit (left: first coronal slice, right: center sagittal slice).

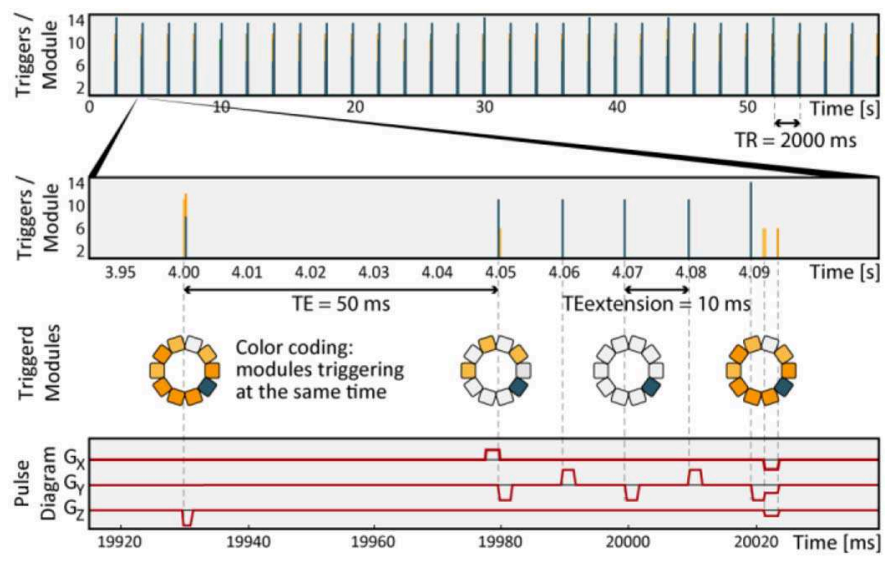

Fig. 15. Triggers/module over time during a gradient echo sequence. On a large time scale TR $=2 \mathrm{~s}$ can be recognized. Zooming the time into one repetition shows the excitation (slice selection gradient) and the 5 echoes being $10 \mathrm{~ms}$ apart starting after a TE of $50 \mathrm{~ms}$. The position and timing of the triggering modules reflect the composition of the MRI sequence.

\section{Advanced Applications}

1) Selective Triggering: The last three columns of Table I show the results of the selective trigger tests. All sequences cause triggers at the standard trigger threshold of $0 \mathrm{mV}$. At $42 \mathrm{mV}$, only the sequences with gradient mode set to "default" or "maximum" trigger. A threshold voltage of $58 \mathrm{mV}$ selects the sequences with a "maximum" gradient mode.

2) Recognition of sequences: The top graph in Fig. 15 visualizes the amount of triggers per module over time during the execution of a $\mathrm{T}_{2} \mathrm{w}$ gradient echo sequence. The relatively long TR of $2 \mathrm{~s}$ is directly visible. Zooming into one repetition reveals the slice selection gradient for the excitation and the first echo at $\mathrm{TE}=50 \mathrm{~ms}$. All five subsequent gradient echoes are detected $10 \mathrm{~ms}$ apart, which matches $\mathrm{TE}_{\text {extension. }}$. The positions, timing, and number of the triggering modules matches the pulse diagram of the sequence (lowest row): Except for three times (out of 156), the echo-generating Y-gradients are only detected by one module; none of the echoes was missed. The 33 phase-en-

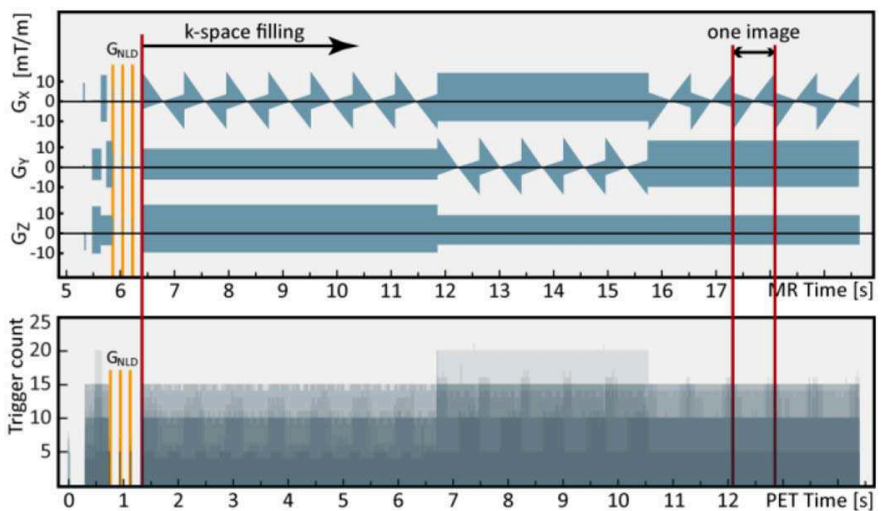

Fig. 16. Pulse diagram (top) of the survey sequence (only end of the preparation phase shown). The trigger count of the gradient sensors are shown with transparency for the different modules (bottom). Clearly recognizable are the three crusher gradients $\left(\mathrm{G}_{\mathrm{NLD}}\right)$ of the noise level determination and the structure of the k-space filling.

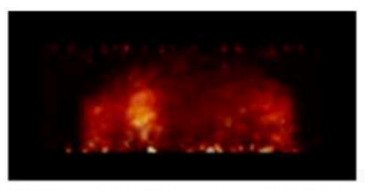

Without motion compensation

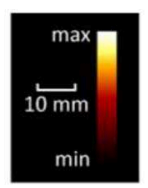

Fig. 17. PET images of a PVA cryogel phantom transformed during the PET measurement according to recorded breathing pattern. Without motion compensation (left) and a gated image based on motion data determined from the concurrent MRI scan.

coding X-gradients are (with one exception) only seen by three other modules; again, none of the gradients was missed by the any of these three modules.

3) Recognition of Sequence Phases: The pulse diagram in Fig. 16 shows the direction and gradient strength of all three gradients at the end of the preparation phase and the beginning of the k-space filling phase of the scout sequence (the exact shape of the gradients is not visible, since the time-zoom-factor is too low). The structure of the sequence with the three orthogonal stacks of images (with seven images for the first stack and five images for the other orientations) are recognizable. The beginning of the k-space filling can thus be determined and the synchronization is done accordingly. The three crusher gradients of the noise level determination (preceding the k-space filling) are also distinguishable. The time between the noise level determination and the k-space filling is slightly longer, than in in the pulse diagram. Furthermore, the length of the receiver optimization phase (preceding the noise level determination) is longer than in the diagram.

\section{Application Example}

Due to the synchronization, the PET list-mode data is directly linked to the taken MR images. The PET data could thus be gated into several bins according to the detected motion in these images. A PET image of the phantom without motion compensation ( $\sim 3.6 \mathrm{MBq}, 9$ minutes acquisition time, 3.7 Mcoincidences, OSMAPOSL reconstruction algorithm of the STIR library [18] with 3 iterations and 7 subsets) and a gated image from $16.11 \%$ of the data, are shown in Fig. 17. 

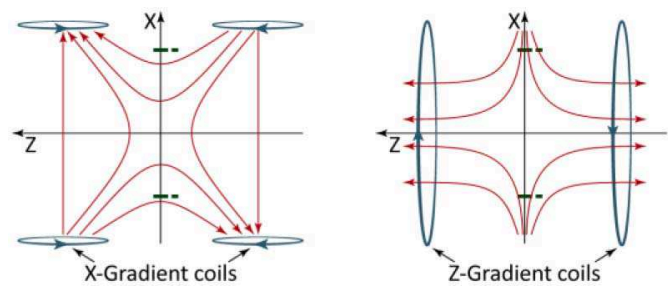

Fig. 18. Sketch of currents in the gradient coils (blue) and the gradient fields in the Y-plane (red) of the X-gradient (left) and the Z-gradient (right). The cross sections of the detector coils are indicated as green lines (longer lines are the transaxial coils). The center of the PET gantry is slightly higher than the axis of the MRI scanner (Z-axis).

\section{DISCUSSION}

\section{A. Sensor Characterization}

1) Threshold Scan: The results in Fig. 10 show that the upper modules of the PET/RF insert trigger earlier than the modules on the bottom. The isocenter of the insert is not the same as the isocenter of the MRI: the PET gantry is shifted $45 \mathrm{~mm}$ upwards. The top modules are closer to the gradient coils, exposed to stronger fields, and thus trigger earlier.

The amount of triggers (from one to three) detected per module corresponds to the theory that the capacitor of the AC-coupling is significantly charged and discharged during the rise time (as described in Section II). It is thus possible to detect positive and negative edges of the switching gradients. The additional fourth trigger at threshold level of $-6 \mathrm{mV}$ can be explained by the discharging curve crossing the trigger level: one time it crosses very slowly and is thus susceptible to noise, supply ripples, and possible ringing.

2) Slew Rate Scans: The plots in Fig. 11 show, that the sensors are much more sensitive to the Z-gradient than to the Xand $\mathrm{Y}$-gradient. Furthermore, the sensitivity to $\mathrm{X}$ - and $\mathrm{Y}$-gradient seems to be similar. This behavior can be explained by the field pattern of the different gradients. Fig. 18 sketches the gradient fields for the Z-gradient and the X-gradient.

Whereas the field lines of the X-gradient are rather parallel to large transaxial gradient detection coil, the Z-gradient fields go right through the coil. This is the reason for the higher sensitivity for the Z-gradient coil. The Y-gradient is similar to the $\mathrm{X}$-gradient, only turned for $90^{\circ}$, which explains the comparable sensitivity in those directions.

Fig. 18 also shows that the isocenter of the PET gantry is shifted $45 \mathrm{~mm}$ upwards. The number of modules, in which a sensor triggers (shown in Fig. 12), reflects this geometric asymmetry: the sensors closer to the gradient coil trigger earlier.

Data points from this experiment were overlaid in the threshold scan of Fig. 11 (lowest slew rate for the Z-gradient, at which at least one sensor triggers). Apart from the value at $0 \mathrm{mV}$ (the first measurement point is at $10 \mathrm{mT} / \mathrm{m} / \mathrm{ms}$ ) the values are coherent. This gives an indication of the reproducibility of the experiments, since the data was not taken at the same day (and the insert was removed from the MRI scanner).

The experiments have shown, that even at very low gradient strengths of $1 \mathrm{mT} / \mathrm{m}$, the sensor triggers reliably at a minimum slew rate of $15 \mathrm{mT} / \mathrm{m} / \mathrm{s}$, which is $7.5 \%$ of the maximum available slew rate. Increasing the number of windings or the area

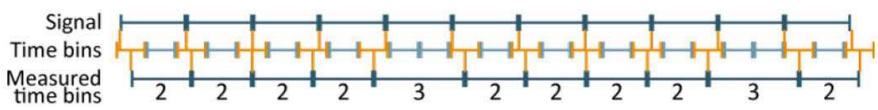

Fig. 19. Example for accumulated time-binning error resulting in repetitive additional counted time bins.

of the coil can even lower that value. The sensor thus triggers reliably at almost every MRI sequence.

3) Time Accuracy and Precision: The measured timing precision of $120 \mu$ s results from the current time binning of $327.68 \mu \mathrm{s}$ (here the deviation is higher than the mean value, since about every $15 \mathrm{~s}$, the accumulated time-binning errors result in an additional counted time bin, see Fig. 19). The lower row of Fig. 13 shows that the time bins could be made smaller to increase the precision. Nevertheless, the required precision of milliseconds for PET motion compensation (Section I-B) is clearly met.

\section{B. Impact on MR Image Quality}

The measured $\mathrm{B}_{0}$-distortion range of $25 \mathrm{~mm}$ is lower than the thickness of the PET detector stack with scintillation crystals, light guide, and two further PCBs (see Fig. 6). It can thus not reach into the field of view. This good result is achieved by the usage of non-magnetic capacitors, which do not contribute to the distortion. The highest distortion is caused by the DAC. If configurable trigger thresholds are not needed, it could be replaced by a voltage divider made from two resistors.

\section{Advanced Applications}

1) Selective Triggering: The three right columns of Table I demonstrate that selective triggering on sequences with high slew rates is possible. With the two tested sequences, it is even possible to choose the triggering sequence by selecting the used gradient mode. Both sequences show a similar trigger behavior since the gradient mode settings result in similar slew rates. Although these are just two sequences, they represent very different applications: TSE sequences are frequently used for anatomical imaging with a high spatial resolution, whereas EPI sequences are used for very fast imaging. They are thus good examples for the combination of sequences, described in Section I-B: multiple short EPI images for motion detection can be interleafed with a long anatomic TSE imaging sequence.

2) Recognition of Sequences: The detected triggers in Fig. 15 show, that it is possible to recognize the timing of MRI sequences, such as demonstrated with the $\mathrm{T}_{2} \mathrm{w}$ gradient echo sequence. The positions, timing, and number of modules that triggered can be compared to the pulse diagram of the sequence (lowest row of Fig. 15). The slice-selecting gradient is a Z-gradient with a high slew rate of $196 \mathrm{mT} / \mathrm{m} / \mathrm{ms}$, which triggers all modules (but the defect one). This makes sense, as the slew rate scans (in Section IV-A2) have shown that the sensors are most sensitive to the $\mathrm{Z}$-gradients. The echo producing Y-gradient (slew rate $=98 \mathrm{mT} / \mathrm{m} / \mathrm{ms}$ ) only triggers one module (blue). The phase encoding X-gradient (directly before the beginning of the echo train with different slew rates depending on the phase encoding step) triggers three additional modules at the negative slope. At the end of the sequence, the phase encoding is undone with a negative pulse, and the same three modules trigger at the same slope. Finally, a dephasing gradient pulse in 
all direction triggers the rest of the modules, since it also contains a Z-gradient. The recognition of timing and composition of MRI sequences can be useful e.g. in retrospective analysis of data (for instance to correlate the deterioration of PET data to certain MRI activity).

3) Recognition of Sequence Phases: The crusher gradients of the preparation phase and the sequence itself are clearly recognizable in the pulse diagram of Fig. 16, which allows the alignment between the two different time bars. The lengths of the different parts of the preparation phase is different from the pulse diagram. Reasons for that are e.g. the undetermined lengths of e.g. iterative optimizations, as explained in the introduction. As the beginning of the k-space filling is recognizable, it allows a synchronization without separation of the preparation phase (see Section III-D). The characteristic pattern of the preceding noise level determination could be used for an automatic recognition. An automatic time alignment would in that case synchronize on the first gradient after the noise level determination, being the first gradient of the k-space filling.

\section{Example Application}

The presented gated image demonstrates that the synchronization between PET and MRI was successful and precise enough for the human breathing application. The work on MRI-based PET motion compensation is ongoing and first results with methods superior to simple gating are presented in $[4]$.

\section{CONCLUSION}

The measurements demonstrate that a gradient detection circuit is reliably able to detect the beginning of a sequence with a precision of at least $120 \mu \mathrm{s}$. With a standard fixed threshold (which could be set by simple voltage divider), the circuit triggers reliable at almost all gradient activity. Using a DAC to set different trigger thresholds, it becomes possible to selectively trigger on sequences, depending their gradient mode setting. Additionally, timings and pulse diagrams of MRI sequences can be recognized. Although, there exist many different MRI sequences, most of them base on the standard spin and gradient echo techniques, which have been used in this paper to test the presented method. Even complicated 3D-spiral or Lissajous trajectories that start with low, continuously oscillating gradients, normally reach high slew rates within a single TR and will thus trigger the sensor. Exceptions might be very exotic sequences, that apply only very small steps in gradient strength, such as zero TE imaging with the SWIFT technique [19]. These would require e.g. a preceding crusher gradient to be detected.

The presented circuit needs very few components, which makes it inexpensive, is simple to realize, and is furthermore helping to maintain $\mathrm{B}_{0}$ homogeneity. Physical connections between PET and MRI systems are not needed, which keeps electromagnetic disturbances low. When used in larger inserts, e.g. for human head or pediatric inserts, the circuits will be closer to the gradient coils and thus even more sensitive. The method works inherently with most MRI sequences, does not require programming of the MRI scanner, and is a vendor-independent solution.

\section{ACKNOWLEDGMENT}

The authors thank Manfred Zinke (retired from Philips Research Aachen, Germany) for his early contribution to the FPGA firmware and testing. Furthermore, they thank Lars Dues for his support in firmware design.

\section{REFERENCES}

[1] M. S. Judenhofer and S. R. Cherry, "Applications for preclinical PET/ MRI," Semin. Nucl. Med., vol. 43, no. 1, pp. 19-29, Jan. 2013.

[2] C. Tsoumpas et al., "Fast generation of 4D PET-MR data from real dynamic MR acquisitions," Phys. Med. Biol., vol. 56, no. 20, pp. 6597-613, Oct. 2011.

[3] G. Soultanidis, J. Mackewn, C. Tsoumpas, and P. Marsden, "PVA cryogel for construction of deformable PET-MR visible phantoms," IEEE Trans. Nucl. Sci., vol. 60, no. 1, pp. 95-102, Feb. 2013.

[4] G. M. Soultanidis, "Demonstration of motion correction for PET-MR with PVA cryogel phantoms," in Proc. IEEE Nucl. Sci. Symp. Med. Imag. Conf. Rec. (NSS/MIC), 2013, pp. M11-70.

[5] B. Weissler et al., "MR compatibility aspects of a silicon photomultiplier-based PET/RF insert with integrated digitisation," Phys. Med. Biol., vol. 59, no. 17, pp. 5119-5139, 2014.

[6] C. Würslin et al., "Respiratory motion correction in oncologic PET using T1-weighted MR imaging on a simultaneous whole-body PET/MR system," J. Nucl. Med., vol. 54, no. 3, pp. 464-71, Mar. 2013.

[7] C. Catana et al., "MRI-assisted PET motion correction for neurologic studies in an integrated MR-PET scanner," J. Nucl. Med., vol. 52, no. 1, pp. 154-61, Jan. 2011.

[8] M. Henningsson, G. Mens, P. Koken, J. Smink, and R. M. Botnar, “A new framework for interleaved scanning in cardiovascular MR: Application to image-based respiratory motion correction in coronary MR angiography," Magn. Reson. Med., vol. 00, no. January, pp. 0-4, Mar. 2014.

[9] P. M. Dueppenbecker et al., "Gradient transparent RF housing for simultaneous PET / MRI using carbon fiber composites," in Proc. IEEE Nucl. Sci. Symp. Med. Imag. Conf. Rec., 2012, pp. 3478-3480.

[10] J. Wehner et al., "PET/MRI insert using digital SiPMs: Investigation of MR-compatibility," Nucl. Instrum. Methods Phys. Res. A, vol. 734, pp. 116-121, Jan. 2014.

[11] J. Wehner et al., "MR-compatibility assessment of the first preclinical PET-MRI insert equipped with digital silicon photomultipliers," Phys. Med. Biol., vol. 60, pp. 2231-2255, 2015.

[12] C. Weirich et al., "Analysis and correction of count rate reduction during simultaneous MR-PET measurements with the BrainPET scanner," IEEE Trans. Med. Imag., vol. 31, no. 7, pp. 1372-80, Jul. 2012.

[13] B. Weissler, P. Gebhardt, M. Zinke, F. Kiessling, and V. Schulz, "An MR-compatible singles detection and processing unit for simultaneous preclinical PET/MR," in Proc. IEEE Nucl. Sci. Symp. Med. Imag. Conf. Rec. (NSS/MIC), 2012, pp. 2759-2761.

[14] P. Gebhardt, B. Weissler, M. Zinke, F. Kiessling, P. K. Marsden, and V. Schulz, "FPGA-based singles and coincidences processing pipeline for integrated digital PET/MR detectors," in Proc. IEEE Nucl. Sci. Symp. Med. Imag. Conf. Rec. (NSS/MIC), 2012, pp. 2479-2482.

[15] B. Weissler et al., "Design concept of world's first preclinical PET/MR insert with fully digital silicon photomultiplier technology," in Proc. IEEE Nucl. Sci. Symp. Med. Imag. Conf. Rec. (NSS/MIC), 2012, pp. 2113-2116.

[16] B. Weissler et al., "A digital preclinical PET/MRI insert and initial results," IEEE Trans. Med. Imag., 2015, to be published.

[17] J. E. Mackewn et al., "PET performance evaluation of a pre-clinical SiPM based MR-compatible PET scanner," IEEE Trans. Nucl. Sci., 2015, IEEE Xplore website, Early Access.

[18] K. Thielemans et al., "STIR: Software for tomographic image reconstruction release 2," Phys. Med. Biol., vol. 57, pp. 867-883, 2012.

[19] D. Idiyatullin, C. Corum, J. Y. Park, and M. Garwood, "Fast and quiet MRI using a swept radiofrequency," J. Magn. Reson., vol. 181, no. 2, pp. 342-349, 2006. 